\title{
Maîtrise des rejets de phosphore dans les effluents d'élevage
}

\author{
F. MESCHY1, C. JONDREVILLE', J.-Y. DOURMAD4, A. NARCY3, Y. NYS \\ ${ }^{1}$ INRA, AgroParisTech, UMR791 Physiologie de la Nutrition et de l'Alimentation, F-75231 Paris, France \\ 2 INRA, INPL-ENSAIA, USC340 Animal et Fonctionnalités des Produits Animaux, \\ F- 54505 Vandoeuvre-les-Nancy, France \\ 3 INRA, UR83 Recherches Avicoles, F-37380 Nouzilly, France \\ ${ }^{4}$ INRA, Agrocampus, UMR1079 Systèmes d'Elevage, Nutrition Animale et Humaine, F-35590 Saint-Gilles, France \\ Courriel : meschy@jouy.inra.fr
}

\begin{abstract}
Au-delà des objectifs de production et de santé, la nutrition animale doit aussi répondre dorénavant aux préoccupations environnementales. Limiter les rejets en phosphore, dont l'excès dans les effluents d'élevage peut conduire à l'eutrophisation des eaux de surface, est devenue une des priorités majeures des recherches de l'INRA.
\end{abstract}

Les recherches sur le phosphore (P) en nutrition animale ont longtemps été focalisées sur l'optimisation des productions et de la reproduction des animaux domestiques. Depuis une quinzaine d'années s'y ajoute une préoccupation environnementale : le phosphore est en effet (avec l'azote) à l'origine de l'eutrophisation des eaux de surface et de ses conséquences (raréfaction de la vie aquatique, odeurs nauséabondes...). L'historique des recherches sur ce thème à l'INRA a été publié dans un numéro spécial d'INRA Productions Animales consacré au phosphore (Guéguen 2005).

A partir des données statistiques Agreste 2005-2006 sur les effectifs animaux en France, on peut estimer à un peu moins de $300000 \mathrm{~T}$ les rejets annuels de $\mathrm{P}$ dans les effluents d'élevage. La figure 1 en indique la répartition selon les différentes catégories d'animaux. Il ne faut pas en conclure que l'élevage est le principal fournisseur de phosphore au milieu ambiant, puisque l'ensemble de l'activité agricole ne représente que $20 \%$ des rejets phosphorés, soit une quantité équivalente aux rejets industriels et le tiers seulement des rejets domestiques (ENGREF 1997). Ces déjections animales représentent une source d'engrais très intéressante pour les plantes mais le déséquilibre, dans quelques régions de l'Europe, entre concentration des élevages hors sol (porcs, volaille et bovins non extensifs) et disponibilité de la surface agricole utile en font une source de pollution pour l'environnement. Ce déséquilibre explique l'importance de la prise en compte des rejets de phosphore liés aux activités d'élevage et l'exploration de toutes les pistes menant à leur réduction. Le coût de traitement de déjections souvent riches en eau et de leur transport vers des zones de production à dominance végétale a conduit à rechercher des solutions locales, d'autant plus que le phosphate est une matière première onéreuse, dont les réserves sont épuisables. La voie nutritionnelle a été logiquement privilégiée par une double approche. La maîtrise des rejets de phosphore passe par une meilleure connaissance des besoins des animaux et de la disponibilité du phosphore des aliments (fourrages, aliments concentrés et sources complémentaires inorganiques), afin d'ajuster le plus précisément possible les apports alimentaires en limitant au maximum les gaspillages inutiles à l'animal et préjudiciables au milieu ambiant. Dans cet article, nous nous limitons volontairement aux aspects nutritionnels.

\section{1 / Porcs et volailles}

La démarche suivie pour limiter les rejets de phosphore par les porcs et les

Figure 1. Origine des rejets en phosphore dans les effluents d'élevages.

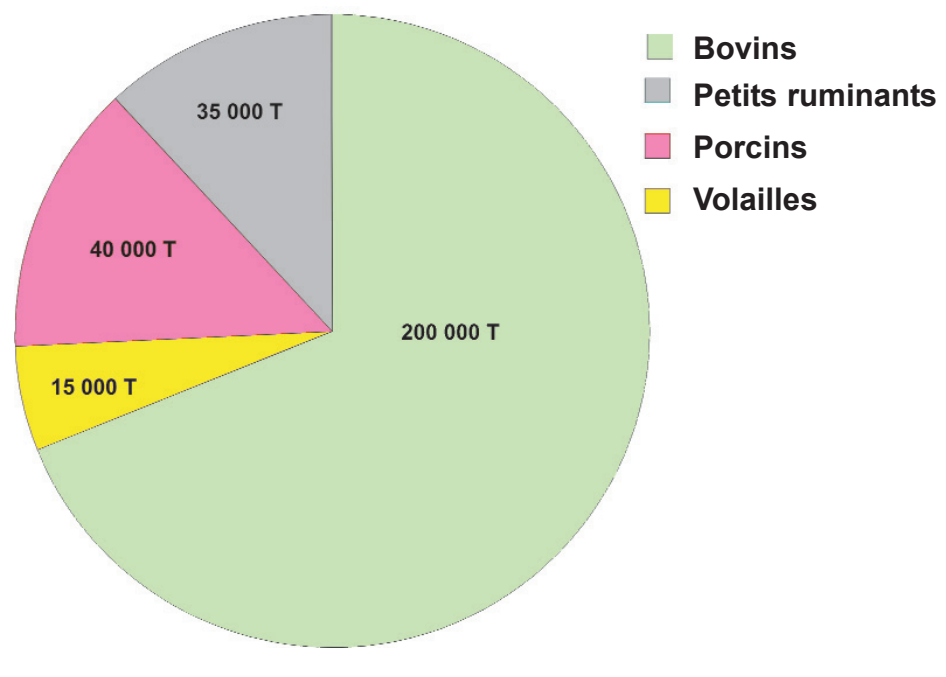


volailles consiste à diminuer les apports alimentaires de cet élément sans compromettre les performances ou la santé des animaux. Elle repose sur la mise en oeuvre de stratégies d'élevage permettant d'ajuster les apports alimentaires de phosphore aux besoins des animaux et de maximiser l'utilisation digestive de $\mathrm{P}$ par les animaux. La recherche a contribué à concevoir de telles stratégies en améliorant les connaissances sur l'utilisation du phosphore des matières premières et des aliments par les animaux et en affinant l'ajustement des apports aux besoins. Cette amélioration n'a été possible que grâce à une meilleure connaissance des besoins des animaux selon leur stade physiologique et leurs performances.

\section{1 / Valeur $P$ des matières pre- mières et des aliments : les tables INRA-AFZ}

\section{a) Caractérisation analytique des matières premières}

Les tables INRA de matières premières publiées il y 20 ans pour les monogastriques (INRA 1989) ont été actualisées récemment. Ces nouvelles tables INRA-AFZ (Sauvant et al 2002, 2004) présentent, pour chaque matière première, la teneur en phosphore total, la proportion de $\mathrm{P}$ phytique pour les matières premières d'origine végétale et l'activité phytasique pour les céréales à paille et leurs coproduits ainsi que pour certaines graines protéagineuses. Cette caractérisation des différentes formes de phosphore est importante chez les monogastriques puisque le phosphore d'origine phytique n'est pas valorisé par les animaux monogastriques qui ne disposent pas de l'équipement enzymatique intestinal nécessaire à l'hydrolyse des phytates. L'utilisation de phosphore d'une matière première d'origine végétale dépend donc en premier lieu des proportions de $\mathrm{P}$ phytique et non phytique dans $\mathrm{P}$ total de la plante. Par ailleurs, les tables indiquent l'activité phytasique dans certaines matières premières, principalement les céréales à paille. Ces matières premières sont en effet dotées d'une activité phytasique intrinsèque permettant l'utilisation de phosphore au moment de la germination. Cette enzyme, en hydrolysant les phytates présents dans les matières premières, contribue à l'amélioration de la digestibilité de phosphore. A l'INRA, après ceux de Sauveur (1989) et Pointillart (1994), les travaux de Frapin (1996), Nys et al (1996), Oury et al (1998) et Jondreville et al (2008) ont contribué à la caractérisation des formes de phosphore et de l'activité phytasique présentes dans diverses matières premières d'origine végétale. Ils ont permis de rendre compte de la variabilité entre et intra matière première pour ces caractéristiques et de l'impact prédominant des facteurs génétiques sur les facteurs environnementaux pour expliquer cette variabilité (Oury et al 1998).

b) Caractérisation de la valeur $P$ des matières premières et des aliments

- Les concepts et les mesures

Dans les tables INRA (1989) la valeur «phosphore» des matières premières correspond à leur teneur en $\mathrm{P}$ total pour les porcs et en $\mathrm{P}$ disponible pour les volailles. En 2002 puis en 2004, les tables INRA-AFZ introduisent la notion de digestibilité apparente chez le porc et conservent la notion de disponibilité chez la volaille. Les animaux utilisés pour évaluer les apports alimentaires de phosphore sont en état de subcarence en $\mathrm{P}$. Chez le porc, on caractérise le comportement de $\mathrm{P}$ alimentaire au niveau du compartiment digestif, alors que chez la volaille, les urines et fèces pouvant difficilement être séparées, on caractérise l'utilisation globale de la source de phosphore étudiée, le meilleur indicateur étant, comme pour les autres espèces, la minéralisation osseuse. Pour estimer la disponibilité du phosphore d'une matière première on compare l'efficacité relative pour la minéralisation osseuse (teneur en cendres du tibia ou des doigts) obtenue avec des doses croissantes de $\mathrm{P}$ provenant de phosphate monocalcique à celle de la matière première à étudier. Pour chacune des deux sources testées, une relation linéaire entre l'apport de phosphore et la teneur en cendres de l'os est établie. La disponibilité est définie comme le rapport entre les deux pentes.

\section{- Valeur $\mathrm{P}$ des matières premières}

Les valeurs présentées dans les tables INRA-AFZ (Sauvant et al 2002, 2004) proviennent pour la plupart de mesures effectuées dans ces conditions sur de jeunes oiseaux à l'INRA (Sauveur 1989, Nys et al 1996, Oury et al 1998). Chez le porc, les valeurs sont issues de mesures effectuées à l'INRA (Pointillart 1994, Dourmad 2002) et s'appuient sur les informations présentes dans la littérature, en particulier sur les travaux menés par Arvalis-Institut du Végétal (Skiba et al 2004). Cette base de données, dans laquelle les teneurs en $\mathrm{P}$ total, $\mathrm{P}$ phytique, activité phytasique et digestibilité du phospho- re étaient renseignées pour 40 lots de matières premières, a permis d'estimer la digestibilité du phosphore phytique et non phytique. Elle démontre également que la phytase végétale permettait de libérer environ $0,5 \mathrm{~g}$ de $\mathrm{P}$ digestible pour 1000 unités de phytase (Jondreville et Skiba résultats non publiés). Ainsi, chez le porc, afin de rendre compte de la dénaturation de la phytase végétale dans les aliments ayant subi un traitement thermique, deux valeurs de digestibilité apparente de phosphore sont proposées : l'une (dP) correspondant à une matière première dont la phytase intrinsèque est dénaturée et l'autre (dPphy) correspondant à la matière première native. Pour les matières premières à activité phytasique significative, comme les céréales à paille et leurs coproduits, dPphy est supérieure à dP. Par exemple, la digestibilité de $\mathrm{P}$ du blé tendre est de $30 \%$ dans un aliment granulé et de $45 \%$ dans un aliment de type farine. Lorsque la matière première présente une activité phytasique négligeable, comme le maïs par exemple, ces deux valeurs sont quasiment égales.

Chez la volaille, les travaux menés à 1'INRA (Sauveur 1989, Frapin et Nys 1994, Nys et al 1996, Jondreville et al 2008) ont permis d'estimer l'efficacité de la phytase végétale en termes d'épargne de phosphates : on estime que 500 unités de phytase végétale sont équivalentes à $0,8 \pm 0,1 \mathrm{~g}$ de $\mathrm{P}$ sous forme de phosphate monocalcique.

\section{2 / Améliorer la disponibilité du phosphore}

a) Apporter des sources de P bien valorisées par les animaux

Dans un contexte de limitation des rejets de $\mathrm{P}$ par les animaux monogastriques, l'utilisation des matières premières et phosphates les plus digestibles devrait être favorisée. Les tables INRA-AFZ (Sauvant et al 2002, 2004) permettent de hiérarchiser les différents phosphates indépendamment de toute considération économique. Les tables font également état de la variabilité de la digestibilité de $\mathrm{P}$ intra phosphate et encouragent une bonne connaissance du produit utilisé, afin d'éviter des apports insuffisants de phosphore ou des rejets excessifs de cet élément.

Par ailleurs, les travaux sur les matières premières ont montré la grande variabilité de la digestibilité de $\mathrm{P}$ entre matières premières (19\% pour le tour- 
teau de tournesol à $90 \%$ pour des coproduits laitiers, en passant par $47 \%$ pour le pois, selon Sauvant et al (2004). La prise en compte de ces données est donc impérative pour réduire les marges de sécurité lors de la formulation des aliments.

\section{b) Utilisation de phytases}

L'introduction de matières premières présentant une activité phytasique intrinsèque élevée est une voie d'amélioration de la disponibilité de $\mathrm{P}$, non seulement de la matière première en question, mais des autres ingrédients constitutifs de la ration (Sauveur 1989, Pointillart 1994, Nys et al 1996, Jondreville et Dourmad 2005). Cependant, elle n'est efficace qu'en l'absence de traitement thermique de l'aliment puisqu'elle est presque totalement inactivée à des températures supérieures à $75^{\circ} \mathrm{C}$, valeur assez classiquement atteinte lors de la fabrication des aliments. D'autres voies d'amélioration de la digestibilité apparente ou de sa disponibilité relative par rapport aux sources minérales de $\mathrm{P}$ doivent être adoptées après traitement thermique.

Pour les aliments granulés, la principale stratégie est l'utilisation de phytases microbiennes. La réponse de l'utilisation de $\mathrm{P}$ à des niveaux croissants de phytase est curvilinéaire et la dégradation de $\mathrm{P}$ phytique avant les sites d'absorption n'excède jamais 60-70\%, même à des niveaux élevés de phytase ajoutée. Chez le porc, les données de la littérature ont permis d'établir que 500 unités de phytase microbienne (3-phytase) sont équivalentes à $0,65-0,75 \mathrm{~g}$ de $\mathrm{P}$ digestible apparent. Chez le poulet, l'introduction de 500 à 700 unités de phytase microbienne améliore l'utilisation digestive du phosphore total de 10 à 12 points (Frapin et Nys 1994) et double celle du phosphore phytique (Lescoat et al 2005). La substitution d'une partie du phosphore minéral de 1 à 1,8 g par un apport de phytase microbienne conduit à une réduction de l'excrétion de phosphore de plus de $40 \%$ (Sauveur 1993, Frapin 1996). Chez le dindon, 1'incorporation de 250 à 500 UI de phytase microbienne économise $0,5 \mathrm{~g}$ de $\mathrm{P}$ du phosphate monocalcique et réduit de $27 \%$ l'excrétion de $\mathrm{P}$ (Juin et al 2001). Enfin, chez le canard, un réduction importante des rejets de phosphore est possible par réduction des apports de phosphore minéral et introduction de phytase microbienne (Bernardet et al 2005)

\section{3 / Estimation du besoin en $P$ des animaux}

Chez le porc comme chez les volailles, le besoin repose sur la maximisation de la minéralisation osseuse et excède celui qui est nécessaire à la maximisation de la vitesse de croissance.

\section{a) Bases d'estimation du besoin en} phosphore des porcins

Le besoin en phosphore des porcs est estimé selon une approche factorielle, qui consiste, pour chaque stade physiologique, à additionner les besoins estimés pour l'entretien, la croissance et la production. Initialement paramétré par Guéguen et Perez (1981), ce calcul permettait d'estimer le besoin en phosphore absorbable réel. La quantité de phosphore alimentaire nécessaire à la couverture des besoins était calculée en divisant le résultat du calcul factoriel par le coefficient d'absorption réelle du phosphore, estimé en considérant que respectivement $30 \%$ du $\mathrm{P}$ phytique et $70 \%$ du P non phytique étaient absorbables. Pour un aliment à base de maïs et de tourteau de soja, couramment utilisé dans l'alimentation du porc à cette époque, le coefficient d'absorption réelle était estimé à $50 \%$.

L'introduction des valeurs de phosphore digestible apparent pour l'estimation de la valeur des matières premières et des aliments a nécessité une adaptation de ce calcul qu'ont explicitée Jondreville et Dourmad (2005). En effet, lorsque l'aliment est caractérisé par sa teneur en $\mathrm{P}$ digestible apparent, les pertes endogènes fécales de phosphore sont déjà comptabilisées dans la valeur qui lui est attribuée. Dans ce nouveau système, le besoin d'entretien est donc égal aux seules pertes urinaires de P. Jondreville et Dourmad (2005) ont également mis à jour l'estimation du besoin pour la croissance, la production de lait et le développement des produits de la conception. Le besoin de phosphore pour la croissance est calculé à partir d'équations de prédiction de la quantité corporelle de phosphore en fonction du poids vif des animaux. Ces équations ont été établies par Dourmad et al (2002) à partir de résultats récents de la littérature. L'exportation de phosphore via le lait est estimée à partir de la quantité d'azote sécrété dans le lait (Noblet et Etienne 1989) et le besoin de phosphore pour la croissance des produits de la conception à partir de l'estimation de l'accrétion protéique (Noblet et al 1985). Sur la base des résultats obtenus au cours de 17 essais destinés à mesurer la réponse des performances de porcs en croissance à des apports croissants de phosphore réalisés en France entre 1992 et 2001, Castaing et al (2003) ont validé les valeurs de P digestible présentées dans les tables INRA-AFZ et les termes de 1'estimation du besoin.

Les équations proposées par Jondreville et Dourmad (2005) permettent donc d'ajuster l'apport alimentaire de phosphore digestible au stade physiologique et aux performances des animaux. Ainsi, elles facilitent de calculer la concentration alimentaire de phosphore digestible nécessaire pour chaque phase lors d'une alimentation biphase, par exemple. Elles permettent également d'évaluer l'impact des performances sur le besoin de phosphore digestible apparent. Par exemple, la diminution de l'indice de consommation de 0,2 point chez un animal en engraissement nécessite l'augmentation de la concentration alimentaire de phosphore de $0,2 \mathrm{~g} / \mathrm{kg}$. Chez une truie en lactation, la concentration alimentaire de phosphore digestible nécessaire est d'autant plus forte que le nombre de porcelets allaités est élevé et que l'appétit de l'animal est faible (pour 11 porcelets allaités, respectivement 3,9 et $2,2 \mathrm{~g}$ P digestible/kg d'aliment pour une consommation quotidienne de 4 et $7 \mathrm{~kg}$ d'aliment).

b) Bases d'estimation du besoin en phosphore des volailles

L'approche factorielle a été proposée pour estimer le besoin des volailles adultes et en croissance par le groupe Européen de Nutrition avicole de la World Poultry Science Association (WPSA) (Sauveur 1985) à partir d'une estimation très simplifiée des coefficients d'utilisation digestive des minéraux. Chez les volailles, on a cependant plutôt privilégié une approche globale selon laquelle le besoin est défini comme étant l'apport de phosphore permettant de maximiser les performances et la minéralisation osseuse. D’une manière générale, ce dernier besoin excède celui correspondant à la maximisation de la vitesse de croissance. Les besoins en phosphore des principales espèces de volaille à différents âges et stades physiologiques ont été déterminés il y a près de vingt ans (INRA 1989). Cependant, l'intérêt de réduire l'apport de phosphore pour les poules reproductrices (Triyuwanta et al 1992) et les poules pondeuses (Nys 2001) a été confirmé. De même, de nombreux travaux ont été réalisés pour 
déterminer la réduction possible de l'apport de phosphore minéral en présence de phytase et ont souligné les possibilités de réduire d'une manière importante cet apport, notamment en période de finition chez le poulet (Bouvarel et al 1997), la dinde (Travel et al 2005) ou le canard mulard (Bernardet et al 2002).

Pour améliorer l'estimation des besoins, une méta-analyse de données représentant plus de 800 observations chez le poulet de chair a permis d'affiner la détermination des besoins et de démontrer l'influence d'interactions (Lescoat et al 2005, Perrot et al 2006). Elle a permis d'une part, de montrer la richesse des données disponibles mais aussi, d'autre part, la difficulté de proposer des lois de réponses globales étant données les interactions fortes entre l'environnement, y compris l'alimentation, et les performances de l'animal. Des travaux se poursuivent au sein de l'INRA pour préciser les besoins, notamment dans des situations proches des conditions actuellement rencontrées en élevage, et pour construire une modélisation du besoin en phosphore (Letourneau-Montminy et al 2007).

\section{4 / En quoi ces avancées per- mettent-elles d'élaborer des solutions concrètes pour dimi- nuer les rejets?}

Dourmad et al (2002) ont élaboré des modèles permettant de calculer le rejet de phosphore en fonction de la quantité de phosphore ingérée et des performances des animaux. Ces modèles ont servi de base aux travaux du CORPEN (2003). Ils ont également été utilisés pour estimer l'impact des travaux de recherche menés au cours des 20 dernières années sur la réduction des rejets de $\mathrm{P}$. Le tableau 1 présente le bilan en phosphore de porcs du sevrage à l'abattage suivant différentes stratégies d'apports alimentaires de phosphore. La prise en compte des résultats de la recherche menée depuis 20 ans (estimation de la valeur des aliments et des besoins des animaux sur la base du phosphore digestible apparent au lieu du phosphore total) permet de réduire les rejets d'environ $38 \%$ (scénario B vs scénario A). L'introduction de l'alimentation biphase permet de réduire encore les rejets de $11 \%$ (scénario $\mathrm{C}$ vs scénario B). Les scénario $\mathrm{D}$, qui représente les références du CORPEN (2003) est proche du scénario C. En considérant que les apports de phosphore digestible peuvent être réduits de $0,75 \mathrm{~g}$ sous forme d'un phosphate présentant une digestibilité de $\mathrm{P}$ de $80 \%$, on estime que l'utilisation de phytase microbienne peut encore permettre de réduire les rejets de phosphore par les porcs de $20 \%$ (scénario E vs scénario C ou D).

Pour les volailles, une démarche similaire a été conduite afin de réactualiser les estimations du CORPEN de phosphore rejeté afin d'optimiser la fertilisation des cultures. Ces estimations sont également un sujet sensible puisqu'elles déterminent à l'échelle d'un élevage les rejets qui peuvent être 1'objet de taxe. La teneur en phosphore déterminée à partir des fumiers ou lisiers est imprécise ; aussi est-il préférable de réaliser un bilan entrantsortant en prenant en compte, d'une part la composition et les ingérés d'aliments, d'autre part la composition des carcasses et le poids des animaux. La composition corporelle en $\mathrm{N}$ et $\mathrm{P}$ a été analysée pour les principales volailles françaises à différents âges (Nys et al 1997, Rouffineau et al 1999) mais également pour les principaux oligoéléments (Mohanna et Nys 1998) ce qui a permis de démontrer le déséquilibre de la composition des déjections par rapport aux besoins des plantes. Cette approche permet par ailleurs de simuler, comme pour le porc, l'impact de modifications des apports alimentaires de phosphore sur les rejets de cet élément à l'échelle d'un animal, d'une exploitation ou d'une région.

Tableau 1. Estimation des rejets de $P$ chez des porcs du sevrage à l'abattage suivant différentes stratégies d'apports alimentaires de $P$.

\begin{tabular}{|l|ccccc|}
\hline & A & B & C & D & E \\
\hline $\mathbf{P}$ (g/kg aliment) & & & & & \\
Porcelet 1 ${ }^{\text {er }}$ âge & 10,4 & 6,6 & 6,6 & 6,8 & 6,1 \\
Porcelet 2 $^{\text {ème }}$ âge & 10,4 & 6,6 & 6,3 & 5,8 & 5,8 \\
Croissance & 6,5 & 4,9 & 4,9 & 4,8 & 4,3 \\
Finition & 6,5 & 4,9 & 4,3 & 4,4 & 3,7 \\
\hline $\mathbf{P}$ ingéré (kg/porc) & 1,91 & 1,38 & 1,28 & 1,28 & 1,12 \\
$\mathbf{P}$ retenu (kg/porc) & 0,518 & 0,518 & 0,518 & 0,518 & 0,518 \\
Bilan de P(kg/porc) & 1,39 & 0,859 & 0,764 & 0,758 & 0,601 \\
Retenu/ingéré (\%) & 0,27 & 0,38 & 0,40 & 0,41 & 0,46 \\
\hline
\end{tabular}

Les porcelets $1 \mathrm{er}$ âge, porcelets 2 ème âge, porcs en croissance et porcs en finition pèsent respectivement 8 à $13 \mathrm{~kg}, 13$ à $30 \mathrm{~kg}, 30$ à $60 \mathrm{~kg}$ et 60 à $110 \mathrm{~kg}$, leur indice de consommation est de respectivement $1,4,1,9,2,5$ et 3,1 .

A : P total (INRA 1989), 1 phase ; B : P digestible (Jondreville et Dourmad 2005), 1 phase ; C : P digestible (Jondreville et Dourmad 2005), 2 phases; D: CORPEN (2003), 2 phases; E : P digestible (Jondreville et Dourmad 2005), 2 phases, Phytase. 
Figure 2. Relation entre l'ingestion et l'excrétion fécale de phosphore chez le ruminant.

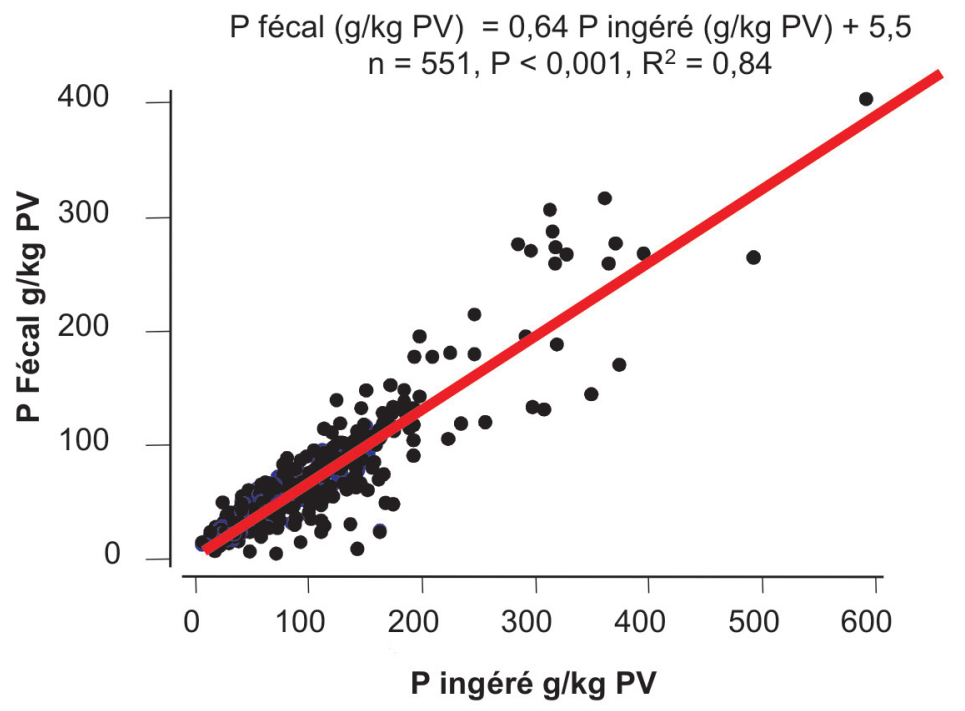

sente l'essentiel de l'apport alimentaire de phosphore (Godoy et Meschy 2001). Les travaux ont donc été centrés sur les points qui semblaient perfectibles, principalement les besoins des animaux, ou plus précisément les apports journaliers recommandés et la valeur nutritionnelle de l'apport alimentaire de P.

\section{1 / Actualisation des apports journaliers recommandés en phosphore}

Les précédentes recommandations d'apport alimentaire en éléments minéraux majeurs (INRA 1988) avait été présentées aux XVIIèmes Journées du Grenier de Theix en décembre 1987 et publiées dans le dernier numéro du Bulletin Technique du CRZV de Theix (Guéguen et al 1987) qui allait être remplacé par INRA Productions Animales. Les recommandations de 1988 étaient basées sur la connaissance de l'époque, il devenait donc indispensable de les actualiser en intégrant la production scientifique disponible depuis 1985 environ.

La définition des apports journaliers recommandés suit une démarche factorielle qui consiste à évaluer d'une part les différentes catégories de dépenses, ou de besoins, (entretien, croissance, gestation et lactation) et, d'autre part à estimer l'efficacité digestive de l'apport alimentaire ou Coefficient d'Absorption Réelle (CAR) qui caractérise la fraction véritablement disponible pour la couverture des besoins. En 1988, faute d'information suffisante à l'époque, une valeur unique de CAR était adoptée pour un stade physiologique donné, ce qui revenait à attribuer une efficacité identique pour l'absorption de phosphore à tous les aliments (et toutes les rations). Des données expérimentales nouvelles nous ont permis d'établir des CAR spécifiques pour les grandes catégories d'aliments destinés aux ruminants. Les apports journaliers recommandés peuvent désormais être exprimés en phosphore absorbable ainsi que l'apport alimentaire : P total de l'aliment x CAR.

\section{a) Le besoin d'entretien}

Le besoin d'entretien en éléments minéraux majeurs est généralement assimilé à la perte fécale endogène minimale éventuellement augmentée de la perte urinaire obligatoire ; son évaluation est compliquée pour le phosphore car la perte endogène fécale augmente linéairement avec la quantité ingérée. Au-delà du strict besoin d'entretien (part incompressible de la perte fécale endogène), il existe une composante excrétoire variable inutile à l'animal et potentiellement préjudiciable à l'environnement.

Pour préciser le besoin d'entretien, nous avons constitué une base de données regroupant uniquement les publications où la perte endogène était mesurée (marqueur radio-isotopique) et n'incluait pas de facteur interférant connu (vitamine D, hormone de croissance...). Après avoir éliminé les travaux où le niveau d'apport de phosphore était excessif, nous disposions de 75 publications représentant 405 observations. Le traitement de cette base de données a permis d'établir une relation forte entre le besoin d'entretien strict de phosphore (BEp en $\mathrm{g} / \mathrm{j}$ ) et la quantité de matière sèche ingérée (MSI en $\mathrm{kg} / \mathrm{j}$ ), la perte urinaire obligatoire étant indexée sur le poids vif (PV en $\mathrm{kg}$ ) aussi bien pour les ovins $(\mathrm{BEp}=0,905 \mathrm{MSI}+0,3$ $+0,002 \mathrm{PV}$ ) que pour les bovins (BEp $=0,83 \mathrm{MSI}+0,002 \mathrm{PV})$. Faute de données suffisantes, il ne nous a pas été possible de proposer une équation spécifique pour les caprins pour lesquels nous utilisons l'équation ovins. La comparaison avec INRA 1988 ne montre pas de modification sensible pour les vaches laitières en production mais il est vrai que «l'effet MSI» était déjà partiellement pris en compte par une majoration du besoin d'entretien en fonction de la production laitière (et donc de la MSI). En revanche, ces équations conduisent à une réduction sensible pour les autres catégories de ruminant pouvant atteindre $30-35 \%$ pour les bovins en fin de croissancefinition. Ces équations, bien qu'établies à partir de jeux de données différents, sont en bonne cohérence avec les publications internationales récentes (NRC 2001, GfE 2003, Valk et Beynen 2003, Sehested 2004).

\section{b) Les besoins de production}

Bien qu'évalués à partir de bases différentes, les besoins de croissance, de gestation et de lactation n'ont pas fait l'objet de profondes remises en cause ; il est vrai qu'il existe depuis assez longtemps un bon accord international dans ce domaine.

Les micro-organismes du rumen ont un besoin spécifique en phosphore (supérieur à celui de l'animal hôte) qui est en grande partie satisfait par le recyclage salivaire ; nous n'aborderons pas ce sujet ici car il n'est pas directement lié au contrôle des rejets en phosphore dans les effluents d'élevage.

\section{2 / Utilisation digestive de l'ap- port alimentaire}

L'objectif était de proposer des valeurs de CAR du phosphore spécifiques à certains aliments ou par grandes familles lorsque le nombre de données était insuffisant. Pour traiter cette question, nous avons ouvert la base de données aux travaux ayant mis en œuvre des méthodes indirectes de mesure de l'absorption réelle (rapport de pente par exemple), sous réserve que la source étudiée réalise un apport significatif de phosphore (en général supérieur à $60 \%$ de phosphore total dans le cas des aliments concentrés). Les valeurs de CAR publiées intègrent une marge de sécurité pour tenir compte de la variabilité des mesures et des réponses de l'animal. 
Les CAR des fourrages sont compris entre 0,60 pour le foin de luzerne et 0,90 pour les pulpes de betteraves surpressées ensilées alors que ceux des aliments concentrés s'échelonnent entre 0,63 pour le tourteau de coton à 0,76 pour l'orge. Il est à noter que ces valeurs sont au moins égales et très souvent supérieures à la valeur de 0,60 retenue en 1988 pour les vaches laitières à haut niveau de production ; il s'ensuit une revalorisation non négligeable de la valeur-phosphore des aliments.

A quelques exceptions près, la satisfaction des besoins en phosphore des ruminants nécessite un apport complémentaire réalisé par des sources inorganiques de P. Il convient de souligner que, chez le ruminant, nous ne retrouvons pas la hiérarchie des phosphates minéraux évoquée pour les porcs ou les volailles. Les phosphates de bonne valeur nutritionnelle (dont l'extraction de phosphore dans l'eau et l'acide citrique à $2 \%$ est supérieure à $90 \%$ ) ne présentent pas de différence significative pour leur utilisation digestive chez le ruminant (Jongbloed et al 2002) et la valeur moyenne de CAR de 0,65 peut être retenue.

La seconde composante de la «valeur-phosphore» des aliments est leur teneur en phosphore total. Pour les aliments concentrés, la mise à jour des concentrations en phosphore (Sauvant et al 2004) ne conduit pas à des différences notables ; en revanche, la composition minérale des fourrages a fortement évolué au cours des 20 dernières années. La mise à disposition de leurs résultats analytiques par nos différents partenaires nous a permis de constituer une base de données regroupant 30000 échantillons récents (moins de 5 ans lors de la constitution de la base en 2004-2005). L'exploitation de cette base de données a permis d'actualiser les valeurs précédemment publiées (INRA 2007). En règle générale, la teneur en phosphore des fourrages est en nette diminution ( $30 \%$ en moyenne), ce qui n'est pas sans conséquences sur l'équilibre des rations.

\section{3 / Conclusion : applications et conséquences}

Les apports journaliers recommandés en phosphore absorbable pour les ruminants ont été présentés aux 9ème Rencontres Recherches Ruminants (Meschy 2002) puis à la 37ème University of Nottingham Feed Manufacturer Conference (Meschy 2003). Les valeurs de CAR du phosphore des aliments concentrés ont été reprises et complétées dans les tables INRA-AFZ (Sauvant et al 2004) et celles des fourrages dans le «Livre Rouge» (INRA 2007). Ces apports journaliers recommandés étendus aux autres éléments minéraux majeurs sont intégrés dans le logiciel de rationnement INRAtion.
Après une période de «rodage» et d'explication pendant laquelle nous avons bénéficié, sur le terrain, de l'aide efficace de nos partenaires professionnels (agricoles et industriels), les apports journaliers recommandés en phosphore absorbable sont adoptés désormais par de nombreux éleveurs. L'application des apports journaliers recommandés et surtout la revalorisation de l'efficacité digestive du phosphore des aliments conduit à une réduction de 15 à $20 \%$ de l'apport de phosphore aux ruminants selon les rations, ce qui correspond à une "épargne» des rejets en phosphore de l'ordre de $25000 \mathrm{~T}$ par an pour les seules vaches laitières.

La diminution de l'apport alimentaire de phosphore chez les ruminants réduit les marges de sécurité excessives des précédents systèmes (faute d'information suffisante à l'époque) et par conséquent le «droit à l'erreur». Pour être efficace et sécurisée, l'application des apports journaliers recommandés en phosphore absorbable doit être accompagnée d'une bonne maîtrise de l'ensemble du système d'alimentation : capacité d'ingestion des animaux, teneur en phosphore des aliments, choix de l'aliment minéral adapté à la ration.

\section{Références}

Agreste, 2005-2006. www.agreste.agriculture.gouv.fr

Bernardet M.D., Nys Y., Guy G., 2002. Détermination du besoin en phosphore chez le canard mulard male durant la phase de croissance et finition. $5^{\text {eme }}$ Journ. Palmipèdes à foie gras, 9-10 octobre, Pau, France, 224-227.

Bernardet M.D., Guy G., Broz J., Nys Y., 2005. Effet de l'apport d'une phytase microbienne (Ronozyme P5000) chez le canard de barbarie sur la rétention de phosphore. 6ème Journ. Rech. Avicole, 30-31 mars, Saint-Malo, France, 226-229.

Bouvarel I., Barrier-Guillot B., Nys Y., Lessire M., Widiez J.L. 1997. Besoins en phosphore disponible et utilisation d'une phytase microbienne chez le poulet de chair en période de finition. Sci. Tech. Avicoles, 19, 13-15.

Bravo D., Meschy F., Bogaert C., Sauvant D., 2002. Effects of fungal phytases addition, formaldehyde treatment and dietary concentrate content on ruminal phosphorus availability. Anim. Fd Sci. Technol., 99, 73-95.

Castaing J., Paboeuf F., Skiba F., Chauvel J., Cazaux J.G., van Milgen J., Jondreville C., 2003. Estimation du besoin en phosphore digestible apparent du porc charcutier : synthèse d'essais zootechniques effectués au cours des dix dernières années, Journ. Rech. Porcine Fr., 35, 47-54.

CORPEN, 2003. Estimation des rejets d'azote, phosphore, potassium, cuivre et zinc des porcs. CORPEN (Ed), Paris, France, 41p.

Dourmad J.Y., Pomar C., Massé D., 2002 Modélisation du flux de composés à risque pour l'environnement dans un élevage porcin, Journ. Rech. Porcine Fr., 34, 183-194.

ENGREF, 1997. www. oieau/eaudoc/suntheses/engref/sevin.htm

Frapin D., 1996. Valorisation du phosphore phytique végétal chez l'oiseau : intérêt et mode d'action des phytases végétales et microbiennes. Thèse Ecole Nationale Supérieure Agronomique, Rennes, France, 134p.

Frapin D., Nys Y., 1994. Rôle des phytases dans l'alimentation des volailles : comparaison de l'efficacité des phytases végétales et microbienne. Journ. Valicentre, 13 décembre, Tours, France, 79-91.

GfE. 2003. Recommendation for the supply of energy and nutrients for goats. DLG-Verlag, Frankfurt am Main, Allemagne, 121p.
Godoy S., Meschy F. 2001. Utilization of phytate phosphorus by rumen bacteria in a semicontinuous culture system (RUSITEC) in lactating goats fed on different forage to concentrate ratios. Reprod. Nutr. Dev., 41, 259-265.

Guéguen L., 2005. La petite histoire du phosphore en alimentation animale : les grandes étapes du demi-siècle. INRA Prod. Anim., 18, 149-151.

Guéguen L., Perez J.M., 1981. A re-evaluation of recommended dietary allowances of calcium and phosphorus for pigs. Proc. Nutr. Soc., 40, 273-278.

Guéguen L., Durand M., Meschy F., 1987. Apports recommandés en éléments minéraux majeurs pour les ruminants. Bull. Tech. CRZV Theix, 70, 105-112.

INRA, 1988. Alimentation des bovins ovins et caprins. Jarrige R. (Ed). INRA Editions, Paris, France, $471 \mathrm{p}$.

INRA, 1989. L'Alimentation des animaux monogastriques : Porc, Lapin, Volailles. Seconde édition révisée, INRA Editions, Paris, France, $282 \mathrm{p}$. 
INRA, 2007. Alimentation des bovins, ovins et caprins. Besoins des animaux. Valeur des aliments. Tables INRA 2007. Editions Quæ, Paris, France, 307p.

Jondreville C., Dourmad J.Y., 2005. Le phosphore dans la nutrition des porcs. INRA Prod. Anim., 18, 183-192.

Jondreville C., Genthon C., Bouguennec A., Carré B., Nys Y., 2007. Characterisation of European varieties of triticale with special emphasis on the ability of plant phytase to improve phytate $\mathrm{P}$ availability to chickens. Brit. Poult. Sci., 48, 678-689.

Jongbloed A.W., Kemme P.A., De Groote G., Lippens M., Meschy F. 2002. Bioavailability of major and trace elements. EMFEMA (Ed) Brussels, Belgique, 118p.

Juin H., Nys Y., Broz J., 2001. Comparative evaluation of two phytase preparations in young tukeys fed a wheat-based diet. Arch. Geflugelk., $65,231-235$.

Letourneau-Montminy M.P, Jondreville C., Pomar C., Magnin M., Sauvant D., Bernier J., Nys Y., Lescoat P., 2007. Meta-analysis of phosphorus utilisation by broilers. $1^{\text {th }}$ Eur. Symp. Poultry Nutr., Strasbourg, France, 117120.

Lescoat P., Travel A., Nys Y. 2005. Lois de réponses des volailles de chair à l'apport de phosphore. INRA Prod. Anim., 18, 193-201.

Meschy F. 2002. Recommandations d'apport en phosphore absorbé chez les ruminants. Renc. Rech. Rum., 9, 279-285.

Meschy F. 2003. Re-assessment of dietary allowances: absorbed phosphorus requirements in ruminants. Rec. Adv. Anim. Nutr., 175-189.

Mohanna C., Nys Y. 1998. Influence of age, sex and cross on body concentrations of trace elements (zinc, iron, copper and manganese) in chickens. Brit. Poult. Sci., 39, 536-543.

Noblet J., Etienne M., 1989. Estimation of sow milk nutrient output. J. Anim. Sci., 67, 3352-3359.
Noblet J., Close W. H., Heavens R. P., 1985. Studies on the energy metabolism of the pregnant sow. 1. Uterus and mammary tissue development. Br. J. Nutr., 53, 251-265.

NRC National Research Council, 2001. Nutrient requirements of dairy cattle. $7^{\text {th }}$ revised Ed., National Academy Press Washington DC, $381 \mathrm{p}$.

Nys Y., 2001. Recent developments in layer nutrition for optimising shell quality. $13^{\text {th }}$ Eur. Symp. Poultry Nutr., Blankenberge, Belgique, 30 septembre-04 octobre, 45-52.

Nys Y., Frapin D., Pointillart A., 1996. Occurrence of phytase in plants, animals and microorganisms. In: Phytase in animal nutrition and waste management. Coelho M.B., Kornegay E.T. (Eds), BASF corporation, 213236.

Nys Y, Guivarc'h F., Chadi M., 1997. Variation de la composition des carcasses de poulets de chair en phosphore en fonction de

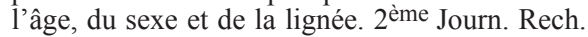
Avicole, 8-10 avril, Tours, France, 177-180.

Oury F.S., Carré B., Pluchard P., Berard P., Nys Y., 1998. Genetic variability and stability of poultry feeding related characters in wheat, in relation to environmental variation. Agronomie, $18,139-150$.

Perrot P., Bouvarel I, Lescoat P., 2006. Réactualisation des références relatives à la composition corporelle en protéines, phosphore et oligo-éléments des volailles : état des lieux et impact sur les rejets avicoles. Sci. Techni. Avicoles, 56, 4-9.

Pointillart A., 1994. Phytates, phytases : leur importance dans l'alimentation des monogastriques. INRA Prod. Anim., 7, 29-39.

Rouffineau F., Guivarc'h F., Nys Y., 1999. Actualisation de la composition corporelle en azote et phosphore des principales volailles de

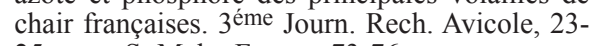
25 mars, St Malo, France, 73-76.

Sauvant D., Perez J.M., Tran G., 2002. Tables de composition et de valeur nutritive des matiè- res premières destinées aux animaux d'élevage : porcs, volailles, bovins, ovins, caprins, lapins, chevaux, poissons. INRA Editions, Paris, France, 301p.

Sauvant D., Perez J.M., Tran G., 2004. Tables de composition et de valeur nutritive des matières premières destinées aux animaux d'élevage : porcs, volailles, bovins, ovins, caprins, lapins, chevaux, poissons. 2ème Edition revue et corrigée. INRA Editions, Paris, France, 301 p.

Sauveur B., 1985. Besoins en minéraux et recommandations d'apport. Rev. Alim. Anim, $389,46-48$.

Sauveur B., 1989. Phosphore phytique et phytases dans l'alimentation des volailles. INRA Prod. Anim., 2, 343-351.

Sauveur B., 1993. Les phytases fongiques dans l'alimentation des volailles. INRA Prod. Anim., 6, 265-267.

Sehested J. 2004. Feeding phosphorus (P) to cattle: a new basis for Nordic recommendations. Acta Agric. Scand., 54, 159-180.

Skiba F., Callu P., Castaing J., Paboeuf F., Chauvel J., Jondreville C., 2004. Variabilité intra matière première de la digestibilité du phosphore des céréales et du pois chez le porc en croissance. Journ. Rech. Porcine Fr., 36, 9-16.

Travel A., Bouvarel I., Aubert C., Chagneau AM, Hallouis J.C., Juin H., Relandeau C., Buttin P., Broz J., Lessire M., 2005. Réduction des rejets de phosphore par voie alimentaire chez le dindon en finition à performances constantes. 6éme Journ. Rech. Avicole, 30-31 Mars, St Malo, France, 343-349.

Triyuwanta C., Leterrier C., Nys Y., 1992. Dietary phosphorus and food allowance of dwarf breeders affect reproductive performance of hens and bone development of their progeny. Br. Poult. Sci., 33, 363-379.

Valk H., Beynen A.C. 2003. Proposal for the assessment of phosphorus requirements of dairy cows. Liv. Prod. Sci., 79, 267-272.

\section{Résumé}

La maîtrise des rejets de phosphore (P) dans les déjections animales passe par une meilleure connaissance des besoins des animaux et de l'utilisation de l'apport alimentaire de P. Les méthodes d'étude sont un peu différentes chez les animaux monogastriques (porcs et volailles) ou les ruminants. En règle générale, la détermination des besoins repose sur une démarche factorielle (estimation des dépenses d'entretien et de production) ; toutefois, une méthode plus globale peut être appliquée chez les monogastriques (optimisation de la minéralisation osseuse). L'intégration des connaissances a permis de préciser les systèmes, l'expression des besoins (et des apports) passant de $P$ total à $P$ digestible apparent (porcs) ou absorbé réel (ruminants). L'évaluation de l'efficacité digestive de l'apport alimentaire de $P$ nécessite, chez le monogastrique, la prise en compte de l'utilisation du phosphore phytique. Cette dernière peut être très différente selon l'activité phytasique propre des constituants du régime, l'utilisation de phytases microbiennes et l'éventuelle dénaturation des phytases lors de la granulation des aliments. Pour ces raisons, deux valeurs de $P$ digestible apparent (activité phytasique intacte ou dénaturée) figurent dans les tables INRA-AFZ; de plus des équations sont proposées pour tenir compte de l'incorporation de phytase microbienne. Pour les ruminants, la compilation des données de la littérature a permis d'établir un coefficient d'absorption réelle de P pour les grandes catégories d'aliments (phosphates concentrés et fourrages). Ces démarches, en permettant une meilleure adéquation des apports aux besoins doivent se traduire par une réduction substantielle des rejets de phosphore dans les effluents d'élevage. 


\begin{abstract}
Control of phosophorus waste in animal droppings

Better knowledge is needed of animal requirements and dietary supply utilisation in order to control phosphorus (P) content in animal wastes. The methods of investigation are quite different in non-ruminant (swine and poultry) and ruminants. The assessment of requirements is generally based on a factorial approach (maintenance and production needing) even if a global method based on bone mineralisation may be performed in non-ruminants. The knowledge available allows to set more precise nutritional standards where requirements (and dietary supply) are expressed in apparent digestible $\mathbf{P}$ (swine) or true absorbed $\mathbf{P}$ (ruminants) instead of total P. In non-ruminants, the evaluation of digestive efficiency of dietary $P$ needs information on phytate $P$ utilisation. This latter may be markedly different following phytasic activity in feedstuffs, addition of microbial phytase and phytasic activity inactivation during feed processing. For these reasons, two apparent digestible $P$ values (with unbroken or destroyed phytasic activity) are in the INRA-AFZ Table; in addition, equations are given to take into account microbial phytase addition. In ruminants, true absorption coefficients have been assessed from literature data for the main groups of feedstuffs. These approaches, which allow a better adequacy between animal requirements and $p$ dietary supply, must lead to a substantial decrease in $P$ run off in animal wastes.
\end{abstract}

MESCHY F., JONDREVILLE C., DOURMAD J.-Y., NARCY A., NYS Y., 2008. Maîtrise des rejets de phosphore dans les effluents d'élevage. INRA Prod. Anim., 21, 79-86. 